\title{
Fast and High-Resolution Ultrasound Pressure Field Mapping Using Luminescent Membranes
}

Simon E. Michels, Mathias Kersemans, Michel Versluis, Guillaume Lajoinie, and Philippe F. Smet*

\section{Accepted version}

This is the authors' version that was accepted for Advanced Optical Materials. The full, edited version can be found at https://doi.org/10.1002/adom.202100085. .

This paper can be cited as:

Michels, S. E., Kersemans, M., Versluis, M., Lajoinie, G., Smet, P. F., Fast and HighResolution Ultrasound Pressure Field Mapping Using Luminescent Membranes. Adv. Optical Mater. 2021, 2100085.

Please contact philippe.smet@ugent.be for further details on this work or visit https://apl.ugent.be/dataAOM for the research data. 


\title{
Fast and High-Resolution Ultrasound Pressure Field Mapping using Luminescent Membranes
}

\author{
Simon E. Michels, Mathias Kersemans, Michel Versluis, Guillaume Lajoinie and Philippe F. Smet
}

S. E. Michels and Prof. P. F. Smet*. Department of Solid State Sciences, LumiLab, Ghent University, Krijgslaan 281, 9000 Gent, Belgium. Center for Nano- and Biophotonics, Ghent University, 9000 Gent, Belgium. *Philippe.Smet@Ugent.be

Prof. M. Kersemans. Department of Materials, Textiles and Chemical Engineering (MaTCh), Mechanics of Materials and Structures (MMS), Ghent University, 9052 Zwijnaarde, Belgium.

Prof. M. Versluis and Dr. G. Lajoinie. Physics of Fluids Group, MESA+ Institute for Nanotechnology, Technical Medical (TechMed) Center, University of Twente, P.O. Box 217, 7500 AE Enschede, The Netherlands.

Keywords: ultrasound, luminescence, visualization, thermoluminescence

\begin{abstract}
Ultrasound is used extensively in medical imaging and therapy, non-destructive testing, flow sensing, underwater range assessment and acoustic microscopy. To ensure the accuracy of these techniques, detailed knowledge of the acoustic pressure field produced by the ultrasonic transducer is required. This paper proposes a functional polymer membrane loaded with ultrasound-activated luminescent microparticles. The semitransparent membrane makes use of the luminescent properties of $\mathrm{BaSi}_{2} \mathrm{O}_{2} \mathrm{~N}_{2}: \mathrm{Eu}^{2+}$ to convert ultrasonic pressure into visible light in a fast and straightforward way, through a process termed Acoustically Produced Luminescence $(A P L) . A P L$ is shown to work within a wide range of acoustic frequencies $(1-25 \mathrm{MHz})$ and pressures (50 kPa - 4.5 MPa), and enables a quantitative characterization of ultrasound fields with a lateral spatial resolution below $200 \mu \mathrm{m}$. At the investigated pressures and frequencies, the light generation mechanism is essentially related to ultrasonic heating rather than mechanical stimulation. These membranes offer effective field mapping possibilities, much faster than conventional time consuming point-by-point hydrophone scanning.
\end{abstract}




\section{Introduction}

Ultrasound techniques allow the investigation of structural details inside (optically opaque) materials and media by sending high-frequency acoustic waves into the investigated medium. Inhomogeneities and interfaces can be mapped and quantified by studying the pressure waves that they scatter or reflect. In particular, this concept is the basis for non-destructive testing, acoustic microscopy and ultrasound imaging. ${ }^{[1-12]}$ Medical ultrasound is the most used imaging modality in modern clinical practice and is gaining popularity in early diagnosis as well. In recent years, ultrasound in combination with microbubbles has shown potency for a broad range of therapeutic approaches such as stem cell therapy, gene therapy, blood-brain barrier opening, local drug delivery and histotripsy. ${ }^{[13-17]}$ The development of hardware for high-speed plane wave ultrasound imaging has paved the road for super resolution imaging, functional imaging, and quantitative imaging ${ }^{[18-20]}$ All these techniques have one common requirement: they need detailed knowledge of the pressure field in order to be fully exploited and ultimately translated to clinical use. Furthermore, recent ultrasonic applications such as acoustic tweezers and particle manipulation also require exact knowledge of (dynamic) beam shapes. ${ }^{[21-25]}$ Two dimensional pressure mapping alternatives for hydrophone scanning exist, e.g. Schlieren imaging, shadowgraphy or thermographic methods, but they offer limited accuracy and are technically challenging. ${ }^{[26-28]}$ For example, Schlieren imaging is affected by out-of-plane waves, difficult to quantify and restricted to visualizing planes orthogonal to the transducer. ${ }^{[26]}$ Recently, thermal imaging was proposed to image pressure fields, but this method suffers from the limitation of thermal cameras in terms of penetration through water for immersed samples and relatively high cost. ${ }^{[28]}$ Therefore, the most common and practical way to quantify an ultrasound field remains a point-by-point scan using a needle hydrophone. This procedure requires physical motion as well as a large number of acquisitions, making it extremely time consuming: a typical scan takes 30 minutes to 1 hour for a limited 2D scan and up to 1 day for a full 3D scan. Furthermore, needle hydrophones require additional specific expert equipment for scanning and data acquisition. Hydrophones are expensive devices, with a cost depending on their 
resolution (ranging from $200 \mu \mathrm{m}$ for piezo-based hydrophones to $10 \mu \mathrm{m}$ for optical hydrophones), yet with a limited lifetime, during which they need to recalibrated frequently.

Here we introduce an innovative material for rapid pressure field quantification using Acoustically Produced Luminescence (APL). Luminescent particles are embedded in a thin polymer sheet, producing a semitransparent acousto-sensitive membrane that can convert ultrasonic pressure into visible light. The polymer membrane is tuned for high acoustical energy absorption and low acoustic reflection by using a thin PDMS (polydimethylsiloxane) slab. The PDMS layer is functionalized by homogeneously loading it with persistent luminescent $\mathrm{Eu}^{2+}$ doped $\mathrm{BaSi}_{2} \mathrm{O}_{2} \mathrm{~N}_{2}$ (BaSiON) microparticles that have the ability to emit green light for over an hour after excitation with blue light or UV radiation. ${ }^{[29-35]}$ This paper presents improvements on our earlier approach to solve the limitations of the proof-of-principle APL system, where the detector sample was fully opaque and not properly tuned for APL. ${ }^{[36]}$ The semitransparent membrane proposed here offers straightforward calibration, ease of use, reliability and high resolution, which enables its use in practice. The APL membrane was used to visualize, within a few seconds, the full cross-section of ultrasound pressure fields with a spatial resolution below $200 \mu \mathrm{m}$ and was shown to work within a wide range of acoustic pressures ( $150-4500 \mathrm{kPa}$ ) and frequencies (1 - $25 \mathrm{MHz}$ ). We also demonstrate how a straightforward calibration allows for accurate and reproducible conversion of the optical images into an absolute pressure field. The membrane provides results that are in good agreement with a calibrated optical hydrophone. These novel functional polymer sheets require little to no expertise and offer a considerable reduction in cost compared to a hydrophone setup, while allowing for a full pressure field mapping orders of magnitude faster than traditional scanning.

\section{Results and discussion}

\subsection{The APL membrane}

The experimental setup used to investigate the APL emission of the membrane is depicted in Figure 1a. The thin APL membrane (Figure 1b-c) is first charged using blue light or UV radiation. When the excitation radiation (a blacklight in this case) is turned off, the membrane continues 
to emit light spontaneously (a phenomenon called afterglow), which is recorded in transmission by a 3.1 MPix monochrome CMOS camera. A typical afterglow curve following a charging period of $30 \mathrm{~s}$ is displayed in solid black in Figure 1d. Upon insonation with an ultrasound wave (in this example a Gymna Pulson 200 physiotherapy transducer was used, operated at $3.3 \mathrm{MHz}$ at a duty cycle of $100 \%$ for 10 seconds), the light intensity rapidly increases and reaches a maximum before slowly decreasing again (red curve in Figure 1d). The APL membrane consists of a translucent polymer sheet with a thickness of $300 \mu \mathrm{m}$ (Figure 1b) embedded with micron-sized BaSiON particles (Figure 1c). The weight proportion of microparticles to polymer is determined by the balance between emission intensity (which increases with a higher particle content) and spatial resolution (which decreases with high particle content due to optical scattering caused by the BaSiON particles) and was determined to be optimal at $20 \mathrm{wt} . \%$ for a membrane thickness of $300 \mu \mathrm{m}$. A micro-CT scan reveals a size distribution of the luminescent particles centered around $36 \mu \mathrm{m}$, with an average distance to the center of the nearest neighbor of $42 \mu \mathrm{m}$ (Figure 1e), indicating that the particles were dispersed relatively homogenously in the PDMS polymer matrix without significant clustering (see the experimental section). PDMS was chosen as the polymer binder material for its low acoustic reflection coefficient in water (acoustic impedance $Z \approx 1.05$ MRayl) and high acoustic absorption $\left(\sim 4 \mathrm{~dB} \cdot \mathrm{cm}^{-1} \cdot \mathrm{MHz}^{-1}\right){ }^{[37]}$ The pressure reflection coefficient for PDMS in water is smaller than $20 \%$, which minimizes interferences with the incoming ultrasound beam as well as the generation of standing waves within the layer. 


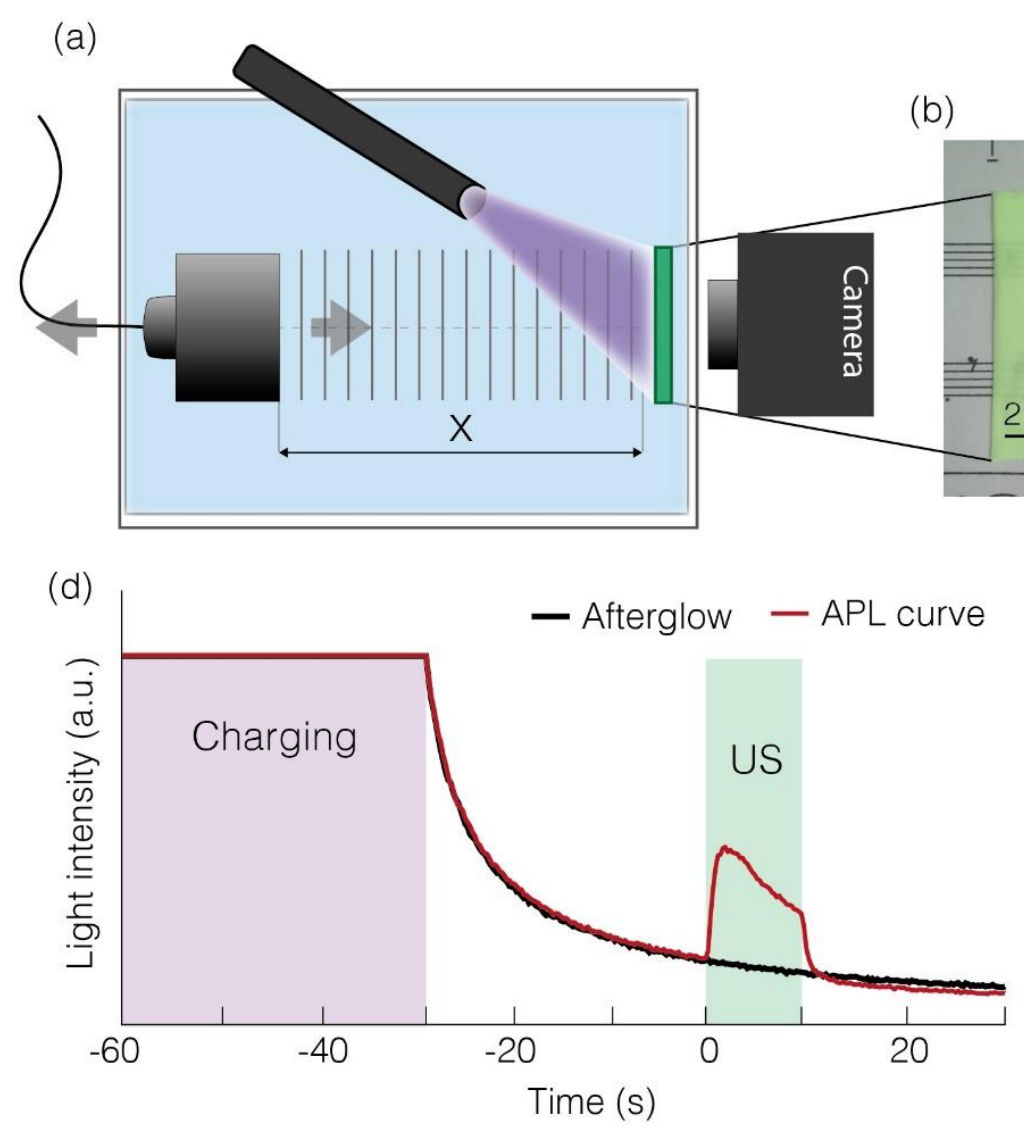

(c)
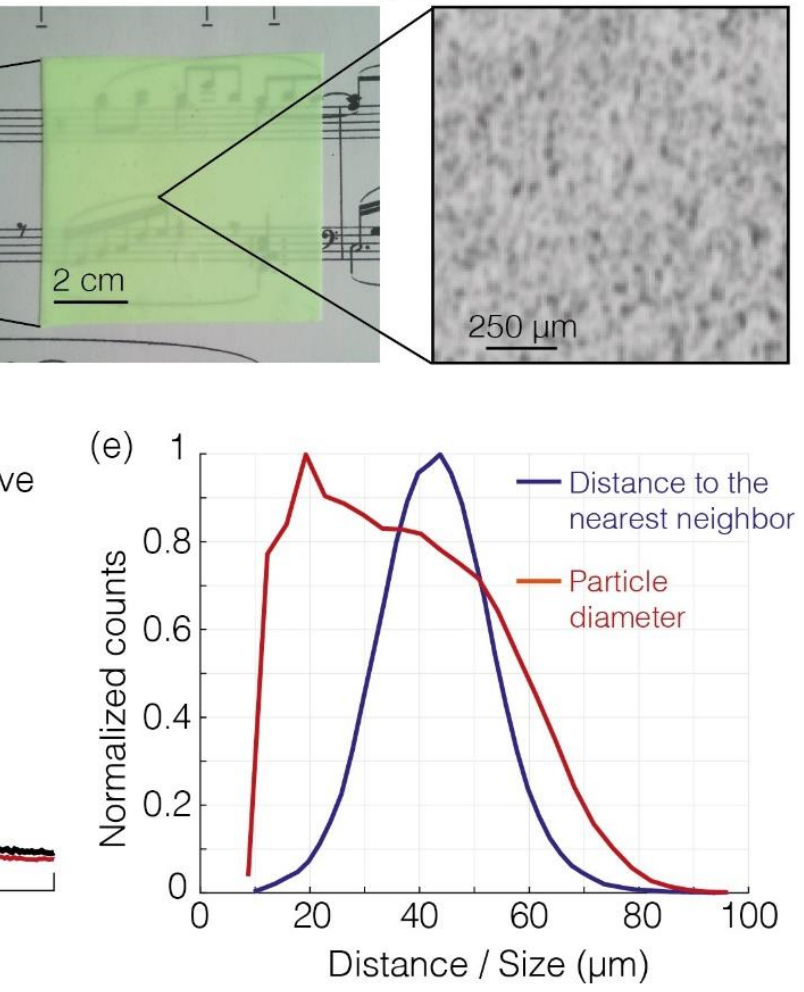

Figure 1: (a) Schematic of the APL setup. Inside a water tank, the transducer can move along the $x$-axis and is aimed at the membrane, which is observed in transmission from outside the tank by a camera. (b) Photograph and (c) X-ray radiographic image of an APL membrane. (d) Example of the APL measurement procedure. The black curve shows the afterglow, which is the decay in emission when the luminescent membrane is left at rest after charging. The red curve shows the increase in emission upon insonation starting at $t$ $=0$ for a duration of 10 seconds. (e) Nearest neighbor distances and diameters of the embedded BaSiON particles, as revealed by a micro-CT scan. 


\subsection{The APL mechanism}

Luminescent materials (also called phosphors) consist of a semiconductor host crystal where some of the atoms are (intentionally) replaced with optically active ions. The ability of certain phosphors to emit light upon insonation with ultrasound has been observed a few years ago and has since been explained as a phenomenon driven by mechanoluminescence $(\mathrm{ML}){ }^{[36,38-44]}$ ML emission occurs when an external pressure or stress deforms the crystal lattice of certain phosphors, thereby inducing the emission of light. ${ }^{[36,41-51]}$ For acoustically produced luminescence, the commonly proposed explanation therefore assumes that the radiation force exerted by the ultrasound beam propagating in the phosphor material induces microstresses, leading to light emission at the location of insonation. One particularly interesting phosphor showing both $\mathrm{ML}$ and acoustically produced luminescence is the oxynitride material $\mathrm{BaSi}_{2} \mathrm{O}_{2} \mathrm{~N}_{2}$ doped with $\mathrm{Eu}^{2+}{ }^{[52-56]}$ This phosphor initially gained popularity as a potential conversion material in white light-emitting LEDs due to its good chemical and thermal stability and efficient conversion of blue light and/or UV radiation into green emission. ${ }^{[57-60]} \mathrm{BaSiON}$ can be effectively excited by electromagnetic radiation with wavelengths between 310 and $460 \mathrm{~nm}$ and emits within a broad wavelength band centered at $494 \mathrm{~nm}$ (Figure S1 of the Supporting Information). During optical excitation, an electron in the $4 \mathrm{f}$ shell of the dopant $\mathrm{Eu}^{2+}$ ion is promoted to the $5 \mathrm{~d}$ shell, both energy levels lying inside the band gap of the piezoelectric ceramic $\mathrm{BaSi}_{2} \mathrm{O}_{2} \mathrm{~N}_{2}$. After crossing a thermal barrier, the electron can eventually be trapped at a meta-stable "trap" level in the band gap (created by e.g. oxygen vacancies or co-dopants). ${ }^{[61-64]}$ With BaSiON now in a charged state (filled electron traps), delayed emission can occur when external energy is supplied to the phosphor. This energy can be the result of electromagnetic radiation, heat, electric fields etc... ${ }^{[65-72]}$ In the case of thermal energy, the delayed emission is called thermoluminescence $(\mathrm{TL})$, and $\mathrm{TL}$ at room temperature is called afterglow. ${ }^{[73-75]}$ For example, this kind of thermally induced light emission can be used for luminescent thermometry. ${ }^{[76-77]}$ In the case of ML, stress applied to a charged BaSiON sample presumably causes the rise of an internal piezoelectric field, which leads to a shift in the energy levels and subsequent release of 
electrons from traps. ${ }^{[45-48,78]}$ In each case, the released electrons are free to migrate back to the (ionized) europium ion where recombination occurs with the accompanying emission of green photons.

In order to determine the dominant mechanism for electron detrapping in BaSiON during an APL event, a charged APL membrane was suspended at an air-water interface. It was then insonated from inside the water by the aforementioned physiotherapeutic piston transducer for 30 seconds and monitored from the air side with a thermographic camera (FLIR A35). When the ultrasound is turned on, a heat profile arises with the distinct near field cross sectional pressure profile of the physiotherapeutic transducer. The recorded thermographic image at IUs $=2.0$ $\mathrm{W} / \mathrm{cm}^{2}$ is shown in Figure 2a. The corresponding pressure map, recorded using a calibrated hydrophone (Onda HGL-0400), is shown in Figure 2b. Both were recorded shortly after the start of insonation, at the moment when the APL emission reached its peak. The temporal temperature profiles, obtained from a small region-of-interest (ROI) in the center of the pattern on the thermographic images, are displayed in Figure 2c, which shows temperature elevations of several degrees Celsius depending on the ultrasound power. Note that this temperature rise is present with or without optical charging of the BaSiON sample. Ultrasonic heating is a wellknown result of the absorption of acoustic waves. For instance, this effect is used as a medical treatment, where High Intensity Focused Ultrasound (HIFU) is able to increase the temperature of tissue up to $85^{\circ} \mathrm{C} \cdot .^{[79-80]}$ 

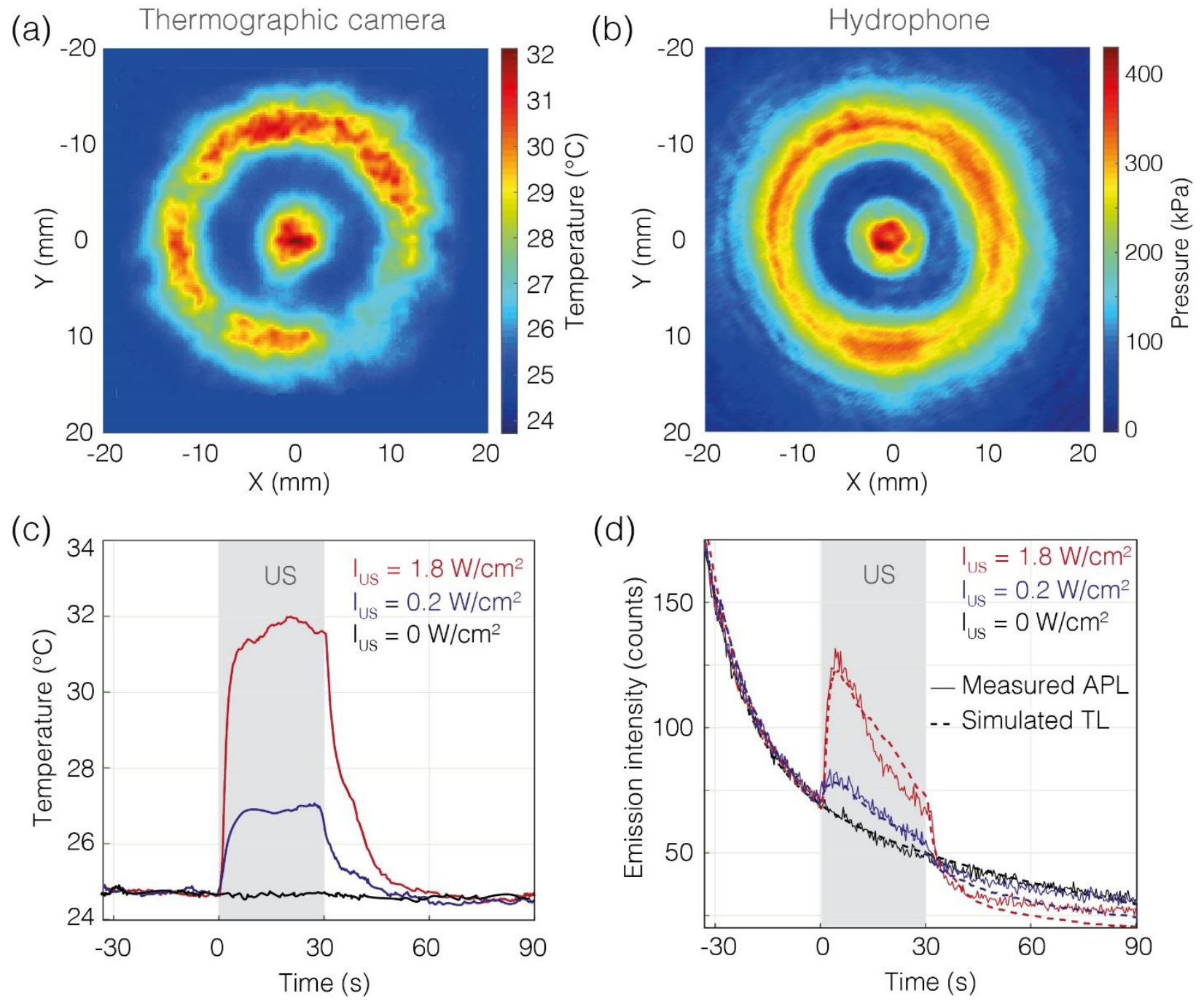

Figure 2: (a) Thermographic recording of the APL membrane at $6 \mathrm{~cm}$ from the surface of a physiotherapeutic transducer. (b) Pressure profile at the same distance from the transducer, as measured with a calibrated hydrophone. (c) Temperature profiles in a small ROI at the center of the profile at different acoustic intensities. (d) Recorded APL intensity and simulated TL intensity for different acoustic intensities. The latter were calculated from the recorded temperature profiles in panel c by using Equation 1.

The temperature profiles shown in Figure $2 \mathrm{c}$ exhibit a saturation (visible by the flattening of the curve 1-5 seconds after the start of insonation) which indicates a balance between the incoming thermal energy and cooling effects (convection and diffusion into the surrounding water and air, diffusion inside the membrane and thermal radiation). Furthermore, Figure $2 a$ and $b$ exhibit 
a good spatial agreement, which is only possible up until the moment when thermal diffusion and convection bring the system into thermal equilibrium (visible from the flattening of the curves in Figure 2c). The thermographic image was recorded at the moment of peak APL emission, which corresponds to the end of the fast linear temperature rise occurring before heat diffusion significantly influenced the temperature distribution (and thus indirectly the APL signal). This phenomenon is also present in thermal imaging of ultrasound fields, where focus sizes are detected that scale linearly with the thickness of the absorber sheet. ${ }^{[28]}$ The small temperature variations on top of the profiles are ascribed to experimentally induced fluctuations (e.g. small disturbances of the membrane during the measurement) rather than to mechanical properties of the transducer or thermal properties of the APL membrane.

A temperature increase due to ultrasonic heating can supply sufficient thermal energy to release trapped electrons in a charged BaSiON particle, leading to TL emission. However, this observation does not exclude possible mechanically induced detrapping. In order to confirm what drives APL light emission (thermally driven $\mathrm{TL}$, mechanically driven $\mathrm{ML}$, or a combination of both), we numerically simulated the expected TL emission of BaSiON. To that end, we have adopted a simple TL model, following first order kinetics (i.e. assuming detrapped electrons are not recaptured in traps but always lead to emission at the dopant). Derived from the RandallWilkins Equations, this model describes the emitted TL intensity as a function of time for a given temporal temperature profile $T(t):^{[81-82]}$

$$
I_{T L}(t)=n_{0} s \sum_{i} \exp \left(-\frac{E_{i}}{k T(t)}\right) \exp \left[-s \int_{0}^{t} \exp \left(-\frac{E_{i}}{k T\left(t^{\prime}\right)}\right) d t^{\prime}\right]
$$

Here $n_{0}\left(\right.$ in $\mathrm{m}^{-3}$ ) is the density of trapped electrons at time $t=0$. $E_{i}$ (in $\mathrm{eV}$ ) denotes the energy barrier for release from a discrete trap level $i$, which is called the trap depth. Note that this constitutes a simplification since persistent phosphors generally host a continuous range of trap level energies. ${ }^{[83-85]} s$ is the so called frequency factor of the trap, which is proportional to the rate (in $\mathrm{s}^{-1}$ ) at which a trapped electron interacts with a lattice phonon and thus "attempts to escape". A standard value of $10^{13} \mathrm{~s}^{-1}$ was used for $s$, for all trap levels. ${ }^{[3,86]}$ In our case, a discrete 
representation with 9 traps provided a satisfactory representation of the afterglow behavior of BaSiON. Note that the $9 E_{i}$ parameters used in this work are not a direct quantification of any physical trap energy levels of BaSiON, but constitute a thermal model of our material. The precise choice of $s$ does not further affect our model since the pseudo energy levels $E_{i}$ are simply fitted to the data. A more detailed description of the model and final parameters is given in section S2 of the Supporting Information. Once the 9 energy levels and their population are determined from the isothermal afterglow curves and the thermoluminescence glow curve (with a fixed heating rate $\beta=0.5 \mathrm{~K}^{-1}$ ) of BaSiON, Equation 1 can be used to calculate the expected thermoluminescent emission when an APL membrane experiences a known temperature rise such as those displayed in Figure $2 c$. Figure $2 \mathrm{~d}$ compares the $\mathrm{TL}$ emission simulated using Equation 1 with the recorded APL emission at different ultrasound intensities, showing a very good agreement. Here, it is important to note that the APL measurements were carried out with a sufficient charging dose to fill all relevant electron traps in the membrane. For example, an 8 W UVA tube with an emission spectrum peaking at $365 \mathrm{~nm}$ placed at a distance of $10 \mathrm{~cm}$ to the sample requires a charging time of approximately 30 seconds. The charging conditions can be changed, as long as the charging wavelength falls within the excitation spectrum of BaSiON, and care is taken to check if the APL membrane is brought to saturation before each measurement, or else, that the charging conditions remain identical for all APL and afterglow measurements which need to be compared. All the curves simulated using the TL model are in excellent agreement with the measured APL curves. In particular, the drop below the natural afterglow curve after the end of the ultrasound excitation $(t>50 \mathrm{~s})$ is well represented. This feature originates from the enhanced trap depletion during insonation as compared to undisturbed afterglow, leading to a lower emission intensity after the ultrasound transducer is turned off. The APL curves of Figure $2 \mathrm{~d}$ show a relatively slow build-up before reaching the peak emission, a build-up time which increases even further for low acoustic pressures. For the class of trap-based mechanoluminescent phosphors to which BaSiON belongs, a mechanical stimulation leads to a very sharp (quasi instantaneous) rise of the emission, provided that the 
pressure threshold (if any) is exceeded. ${ }^{[78]}$ These observations point at different underlying mechanisms. Furthermore, an experiment where the APL membrane was thermally cleaned after charging (by heating up to temperatures between $20^{\circ} \mathrm{C}$ and $50^{\circ} \mathrm{C}$ ) showed the expected APL response when one assumes APL has a thermal nature, and this response is well reproduced by the TL model (Figure S3 of the Supporting Information). Furthermore, extending the delay time between ending the excitation and the insonation to 10 hours does not lead to APL with measurable intensity. In contrast, it was reported earlier that the mechanoluminescence in BaSiON partly arises from much deeper traps. ${ }^{[78]}$ These findings, together with the good match

of Figure $2 \mathrm{~d}$, give a strong indication that, at the investigated pressures and frequencies, ultrasonically generated luminescence in BaSiON is essentially driven by ultrasonic heating leading to a thermoluminescence response, and not by a stress-related piezoelectric effect. Note that this kind of persistent luminescence induced by ultrasonic heating should not be confused with other kinds of ultrasonically induced light generation mechanisms such as "ultrasound-switchable fluorescence". ${ }^{[87-88]}$

\subsection{Frequency dependency of APL visualization}

Measuring a thin cross-section of a pressure distribution inherently requires a thickness of the active phosphor layer that is less than the acoustic wavelength in order to avoid averaging information over the thickness direction. Therefore, in order to test the potential of the APL material to react to a broad range of frequencies, we have fabricated a second type of membrane consisting of a double PET polymer foil enclosing a thin layer of luminescent BaSiON particles ( $\sim 50 \mu \mathrm{m}$ of phosphor layer, see experimental section). This allowed us to investigate APL emission up to an ultrasonic frequency of $25 \mathrm{MHz}(\lambda u s=60 \mu \mathrm{m})$. Figure $3 \mathrm{e}$ shows a schematic overview of the experiment. The sample was insonated by a single element transducer at an incident angle of $30^{\circ}$ in order to minimize ultrasound standing waves. The resulting APL emission was then recorded by a 1.4 MPix monochrome CCD camera (LM165M, Lumenera) through a $2.5 \times$ microscope objective. The APL emission is easily measured with this standard equipment, even at frequencies at the edge of the medical ultrasound range, as shown 
in the inset for $f=25 \mathrm{MHz}$. Thus, APL can be used to visualize the acoustic fields produced by a wide range of ultrasound transducers. Figure 3a-d shows microscopic APL images of the PET membrane at four different ultrasound frequencies and various pressures. APL emission was measured as a function of pressure at 6 ultrasound frequencies (generated by 5 different focused single element transducers). The total insonation duration was 1 second and was applied in repeated bursts of 1000 cycles with a pulse repetition frequency of $10 \mathrm{~Hz}$. The result is shown in Figure $3 f$.

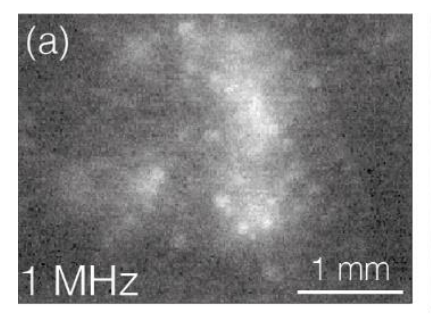

(e)
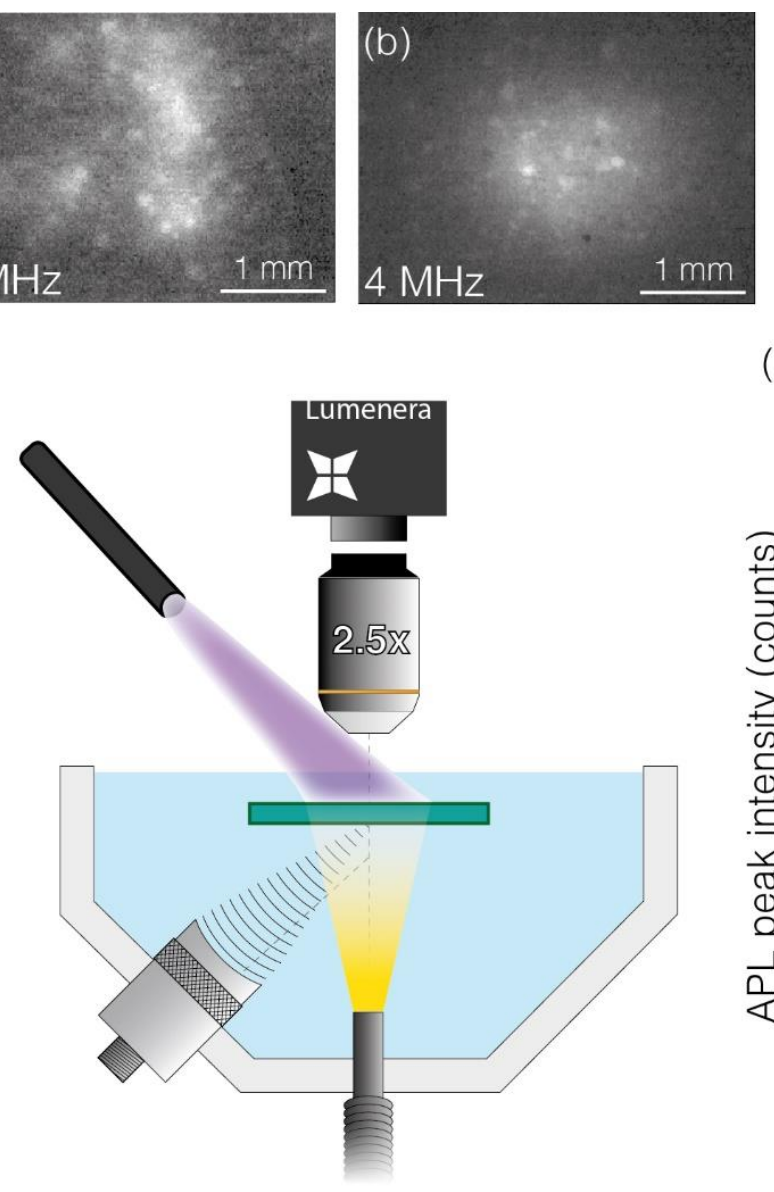
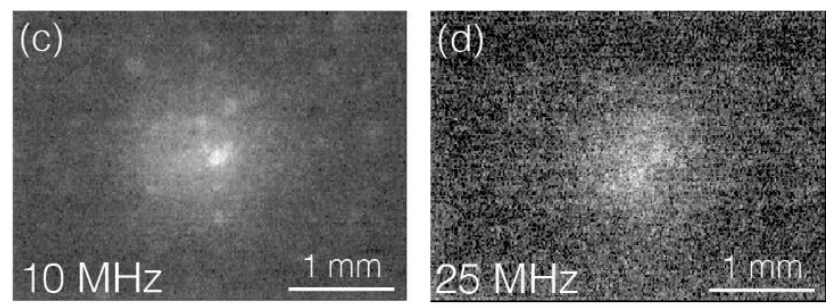

(f)

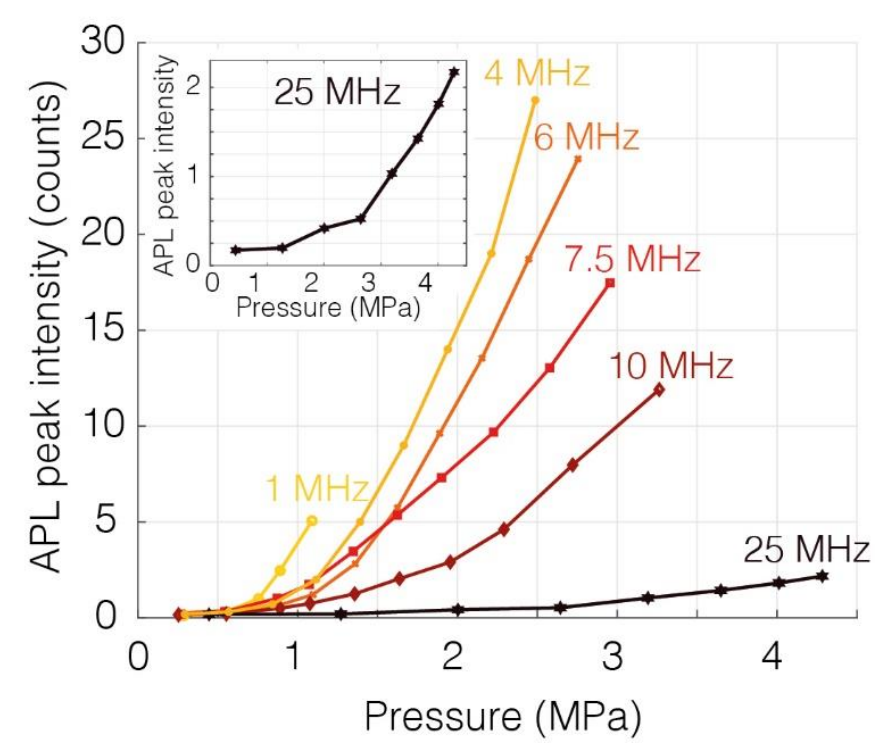

Figure 3: (a)-(d) Normalized microscopic APL frames at different ultrasound frequencies, as recorded with a PET-type membrane. (e) Setup for microscopic observation of APL. The transducer is aimed obliquely at the membrane, which is observed in transmission through a microscope objective. White backlight was used for 
focusing and aligning. (f) APL peak intensities for different ultrasound frequencies and pressures. The inset shows a zoom of the APL response at $f=25 \mathrm{MHz}$.

No general conclusions regarding the dependency of APL on the ultrasound pressure or frequency can be drawn from Figure $3 f$ because of the angle dependent transmission of the ultrasound waves through the PET-type membrane, which is less than optimal at $30^{\circ}$ (see Section S4 of the Supporting Information). For the current purposes however, Figure 3 clearly demonstrated that the APL phenomenon works at a broad range of acoustic frequencies and pressures. While a knowledge of the APL dependency on ultrasound frequency would be interesting, it requires further fundamental research into the frequency dependent absorption of acoustic energy, diffusive and convective heat transfer inside the membrane, etc. Dependency on the ultrasound pressure at $f=5 \mathrm{MHz}$ is described in the next section. One clear benefit of APL imaging over direct thermal imaging is that the emission occurs in the visible region, allowing for visual inspection, as well as the use of a common, off-the-shelf camera. APL visualization also does not suffer from difficult color calibration, which hampers methods using the thermochromic effect of liquid crystals. ${ }^{[89-90]}$ These methods also have a restricted measurement range (depending on the type of liquid crystal), while one APL membrane can be used repeatedly for a wide range of ultrasound frequencies and pressures (Figure 3f). On the other hand, APL does not provide phase information of the ultrasound beam, which can be obtained with hydrophone scanning. Furthermore, it should be noted that the heat increase induced by acoustic absorption depends on the acoustic frequency, and thus on the harmonic content of the ultrasound wave. The absolute APL intensity can therefore be affected by nonlinear propagation of the ultrasound wave in water, for instance when the experiment involves detection at long propagation distances and relatively high pressures for an unfocussed beam. Besides nonlinearity of the propagating wave, additional nonlinearities could arise inside the membrane, considering the luminescent particles that are suspended in the polymer may have some degree of freedom to move. A degree of harmonic content could result in an overestimation of the received pressure, and it is difficult to compensate for this possible 
error since APL does not directly provide information on the frequency content of the pulse, but rather responds to the total acoustic intensity over the entire frequency bandwidth of the ultrasound wave.

\subsection{Quantitative acoustic pressure field mapping}

The PDMS membrane was calibrated by recording the ultrasound pressure field at a distance of $10 \mathrm{~cm}$ from an unfocused $5 \mathrm{MHz}$ transducer (GE H5K, $10 \mathrm{~mm}$ aperture diameter, driven in harmonic continuous wave mode) at different amplified driving voltages, both via APL visualization and needle hydrophone (Onda HGL-0400) scanning (Figure 4a and b, respectively). The optical charging dose was sufficient to bring the BaSiON particles into saturation (all traps filled) and was stopped 30 seconds before the start of insonation. APL pressure quantification was then based on the image recorded by a 3.1 MPix monochrome CMOS camera at the moment of peak emission, before heat diffusion could distort the spatial features of the APL signal.
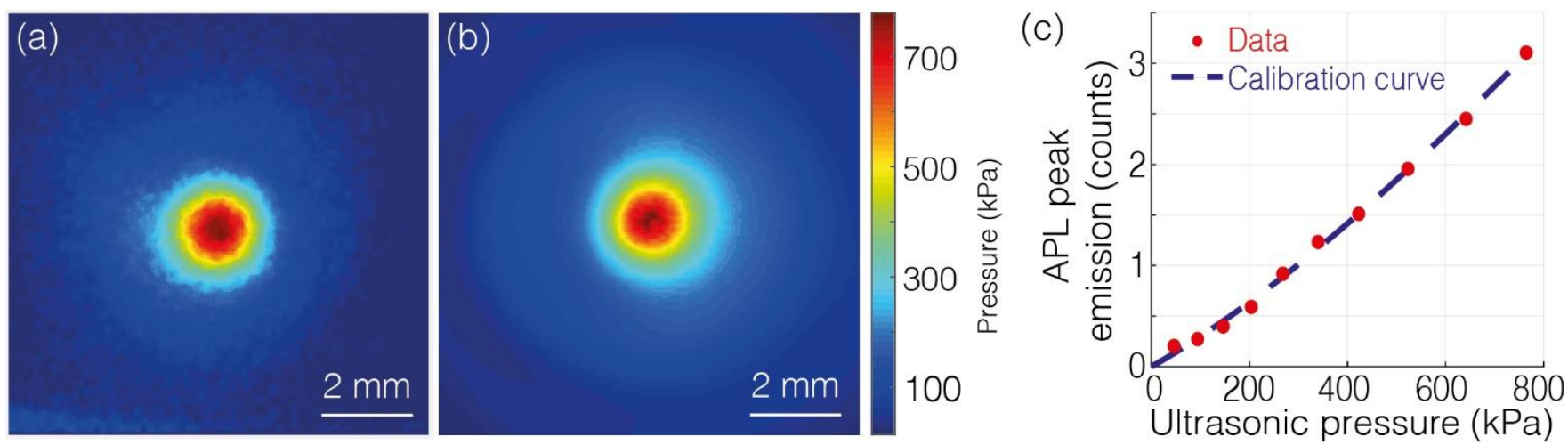

Figure 4: (a) Calibrated APL recording of the pressure field at $10 \mathrm{~cm}$ from the surface of an unfocused $5 \mathrm{MHz}$ transducer. (b) Hydrophone recording of the same field. (c) The calibration curve, obtained through a polynomial fit $\left(R^{2}=0.9978\right)$.

The measured APL emission was normalized by the afterglow to provide a calibration that is independent of the camera used for optical imaging. Thus, an APL membrane only needs to be calibrated once for each new experimental setup, since correcting by the afterglow (recorded after charging saturation) ensures an independence of most experimental conditions (such as 
inhomogeneous material loading, variations in the environmental temperature and the degree of charging). On the other hand, this work pertains to a full water environment of the APL membrane, which covers most of the ultrasound applications of interest (i.e. medical applications). If this environment is changed to other media, different heat transfer dynamics will change the temperature rise in the membrane for an equal amount of deposited ultrasound energy. For both visualization methods (hydrophone and APL), data was collected by averaging over a small region of interest at the center of the pattern (circular ROI with $r \approx 150 \mu \mathrm{m}$ ), which reduced the noise in the APL recording. Doing this for different driving voltages results in a list of peak APL intensities at different acoustic pressures. The final calibration curve, resulting from a third order polynomial fit to this data, is shown in Figure 4c. More details on the calibration procedure and curve can be found in Section 5 of the Supporting Information, and the harmonic content of the ultrasound beam is given in Section 6 of the Supporting Information. The calibration curve is a property of a specific membrane composition, namely a given polymer (thickness) and phosphor material, here PDMS-BaSiON. For a given membrane, the calibration remains dependent on the ultrasound frequency due to frequency-dependent attenuation. Besides these restrictions on the universal applicability of any one calibration curve, further use of Equation 1 could practically remove the need for a precise timing between charging the membrane and turning on the ultrasound since it directly relates the light intensity to the temperature rise (and thus to the local pressure) at any time during the afterglow.

\subsection{Spatial resolution of APL}

For assessing the spatial resolution of the APL visualization method, an L7-4 clinical linear array (ATL, Philips) was used in combination with a Verasonics 256 Vantage system to produce differently sized ultrasound beams. The elevation focus of the array was fixed, while the in-plane focus could be set. The scanner was programmed to successively emit four focused waves with increasing focal depth, the depth of the first focus coinciding with the elevation focus. In the cross-sectional plane, this resulted in four beam spots, with decreasing size (Figure 5a-b). A frequency of $6 \mathrm{MHz}$ was chosen for the ultrasound waves, which is at the edge of the effective 
bandwidth of the L7-4, in order to produce small ultrasound foci and test the resolution limit of APL. Each pulse lasted for 1 second and consisted of 3 bursts of 30 sine waves. The first two frames displaying APL after the beginning of the sonification were used to quantify the maximum resolution of APL. This corresponds to an integration time of the APL emission of 264 ms. Note that choosing an APL frame during the initial fast temperature rise (rather than at the moment of peak emission) provides the best spatial resolution at the cost of a reduced accuracy in pressure quantification (due to a smaller signal-to-background ratio between the APL and afterglow emission). As the calibration curve of Figure $4 c$ was constructed for ultrasound at a frequency of $5 \mathrm{MHz}$, a small error is introduced in the calibration of the field from the L7-4 array, at $6 \mathrm{MHz}$. However, it is expected that this will not overly influence the spatial features of the APL pattern. The obtained APL pressure map (displayed in Figure 5a) was then compared to a hydrophone scan performed at the same distance from the linear array. The hydrophone scan, performed with a step size of $75 \mu \mathrm{m}$, is displayed in Figure $5 \mathrm{~b}$.
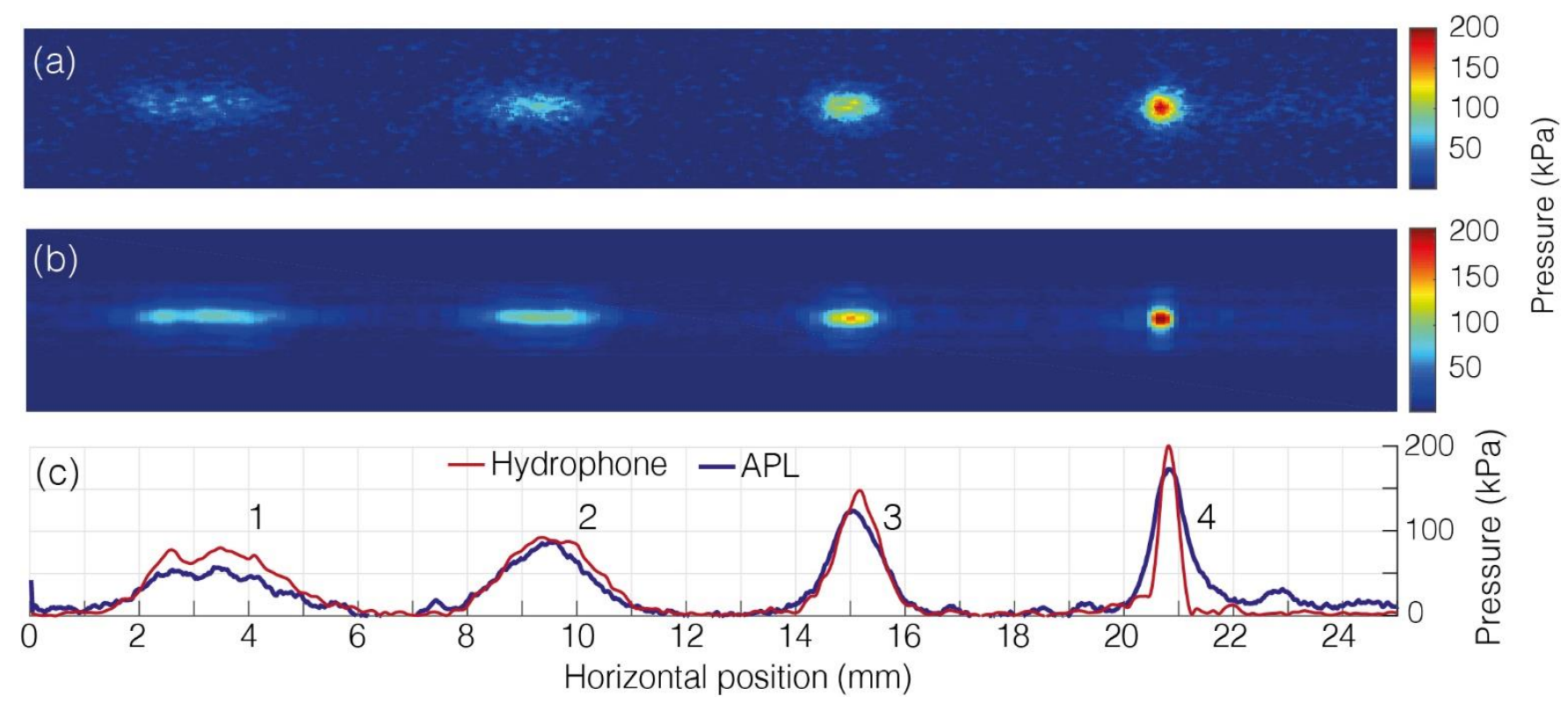

Figure 5: (a) Calibrated APL image of the pressure field of a phased array. (b) Hydrophone recording of the same field. (c) Horizontal cross sections through recordings a and b. The APL cross section was smoothed by using a moving average filter with a span of $47 \mu \mathrm{m}$. 
With a tip size of the needle hydrophone of $10 \mu \mathrm{m}$, we consider the optical hydrophone scan sufficiently accurate to detect the spatial features of the spots, without spatial averaging. From Figure $5 c$, it is clear that the spatial profile of the APL method seems accurate for spots 1, 2 and 3. For the smallest spot 4, however, the cross sectional profile of the APL method appears visibly wider than that of the hydrophone scan. As thermal diffusion is largely avoided in Figure 5a by integrating only the first $264 \mathrm{~ms}$ of the APL signal, this is likely related to the optical scattering from the BaSiON particles in the PDMS layer. In order to assess the resolution loss with APL as compared to the needle hydrophone, the hydrophone profile of spot 4 from Figure $5 c$ was convoluted with Gaussian profiles with increasing width. The best agreement between convoluted hydrophone profile and APL profile was reached (in both horizontal and vertical directions) for a Gaussian width of $170 \mu \mathrm{m}$ (see Section S7 of the Supporting Information).

Finally, a comparison can be made between APL and conventional hydrophone scanning for quantifying ultrasound pressure fields. Using APL, a cross-sectional measurement can be performed in seconds, whereas one 2D hydrophone scan typically takes 30-60 minutes. Highresolution hydrophone scans are thus very limited in size by necessity. The latest fiber optic hydrophones have a flat response to frequencies from $1 \mathrm{MHz}$ up to around $150 \mathrm{MHz}$. Lower frequencies can be reached with different types of hydrophones at the cost of a lower resolution (> $200 \mu \mathrm{m}$ ) and increased fragility. We have shown that one single APL membrane offers a similar performance and responds to ultrasound frequencies between 1 and $25 \mathrm{MHz}$ (at least) and pressures between 50 and $4500 \mathrm{kPa}$. From fundamental considerations, we expect no frequency detection limit, as long as acoustic energy is being absorbed in the membrane. However, in practice, APL emission caused by a very small rise in temperature due to ultrasonic heating (estimated at less than $0.5{ }^{\circ} \mathrm{C}$ under the present observation conditions) will be difficult to detect from outside the membrane, resulting in a practical detection limit on APL, determined by the sensitivity of the optical imaging system. The pressure detection limits of an APL membrane depends on the trap distribution of the luminescent material. As long as this material hosts a sufficient amount of shallow traps, it is thus unlikely that APL emission has a pressure 
threshold: any temperature rise on top of the room temperature should cause an increase in luminescence (see Equation 1). The theoretical upper detection limit is reached when the acoustic pressure is high enough to deplete all the traps almost instantly. However, for BaSiON, this is only expected at pressures (and thus induced temperatures) well beyond the normal operating regime of ultrasound transducers. If required, APL membranes with a higher trap density and with thermally deeper traps can be easily fabricated). The bottleneck for APL detection will therefore be the optical detection equipment. The operating temperature, as it does for hydrophones, will have an influence on the calibration and detection limits of an APL measurement. However, by using a luminescent material with the right trap distribution, the composition of an APL membrane can be tuned to work well outside the operating range of fiber optic hydrophones $\left(5-95^{\circ} \mathrm{C}\right)$. Spatial features of a pressure field can be detected by an APL membrane with a resolution of about $200 \mu \mathrm{m}$, which is larger than the more expensive fiber optic hydrophones but sufficient for most ultrasound fields. Finally, hydrophones are notably fragile instruments, easy to damage through either operator error or cavitation damage. An APL membrane offers a more robust alternative.

\section{Conclusion}

The APL visualization technique employs a semitransparent luminescent membrane which converts acoustic pressure into visible light, allowing for a fast, easy and accurate visualization of an ultrasound pressure field. Modeling the thermoluminescence response showed that, at the investigated pressures and frequencies, APL emission is essentially driven by a thermal release of trapped electrons induced by ultrasonic heating, rather than an $\mathrm{ML}$ based driving mechanism as proposed by earlier studies. The insights presented in this paper allow the fabrication of dedicated APL sensing membranes with thermal and optical behavior tailored to ultrasound field quantification. After a straightforward calibration procedure, APL allows for quantitative determination of the acoustic field produced by a wide range of transducers, with pressures between $50 \mathrm{kPa}-4.5 \mathrm{MPa}$ and frequencies between at least $1-25 \mathrm{MHz}$. An APL membrane only needs to be calibrated once, since correcting by the afterglow ensures an 
independence of the optical setup used. The spatial resolution of a PDMS+BaSiON membrane, consisting of $\mathrm{BaSi}_{2} \mathrm{O}_{2} \mathrm{~N}_{2}: \mathrm{Eu}^{2+}$ (thermo)luminescent particles embedded in a PDMS membrane, was established at $170 \mu \mathrm{m}$. APL visualization shows great promise both for fast screening and full spatial characterization of ultrasound fields in a much faster way than based on hydrophone scanning, increasing the applicability of novel ultrasound techniques that require detailed knowledge of the beam profile.

\section{Experimental section}

\section{Sample preparation}

$\mathrm{BaSi}_{2} \mathrm{O}_{2} \mathrm{~N}_{2}: \mathrm{Eu}^{2+}$ powders were synthesized using a solid state reaction method. Stoichiometric amounts of the chemical precursors $\mathrm{BaCO}_{3}\left(99.95 \%\right.$, Alfa Aesar), $\mathrm{Si}_{3} \mathrm{~N}_{4}$ ( $\alpha$-phase $99.5 \%$, Alfa Aesar) and $\mathrm{EuF}_{3}(99.5 \%$, Alfa Aesar) were thoroughly ground and mixed in an agate mortar. The mixture was then heated to $1425^{\circ} \mathrm{C}$ in a tube furnace and kept at that temperature for 4 hours under a flow of forming gas $\left(10 \% \mathrm{H}_{2}, 90 \% \mathrm{~N}_{2}\right)$. Afterwards, the compound was lightly ground into micrometer sized powder, sieved (mesh no. 270, nominal opening of $53 \mu \mathrm{m}$ ), and inspected for phase purity by $X$-ray diffraction on a $\theta-2 \theta$ Siemens D5000 diffractometer ( $40 \mathrm{kV}, 40 \mathrm{~mA}, \mathrm{Cu}$ $K \alpha$ radiation). PDMS membranes were produced by thoroughly mixing the powder in a 1:4 ratio with PDMS resin. The curing agent was then added, and the mixture was stirred. Finally, the mixture was drop-casted on a PMMA substrate. Thickness was controlled by the razor blade method. The molt was left in a dry stove at $50{ }^{\circ} \mathrm{C}$ for $24 \mathrm{~h}$. This produced a semi-transparent composite PDMS+BaSiON membrane with a thickness of $300 \mu \mathrm{m}$. For the investigation of frequency dependency, PET membranes were produced by spreading the BaSiON powder on a PET foil substrate (125 $\mu \mathrm{m}$ thick) covered with a layer of contact glue. Two identical foils were prepared, the excess powder was tapped off and the two sides were glued together to form a submersible foil sample with a surface loading of BaSiON micropowder of approximately 3 $\mathrm{mg} / \mathrm{cm}^{2}$. 


\section{Measurement setups}

For the thermographic experiments (section 2), a physiotherapeutic Gymna 200 piston transducer was used to produce ultrasound waves with a frequency of $3.3 \mathrm{MHz}$ and acoustic output powers between 0.1 and $2.0 \mathrm{~W} / \mathrm{cm}^{2}$. The resulting APL emission was recorded by a Ximea MC031MG-SY-UB camera equipped with a Sony CMOS sensor chip (3.1 MPix) and an Edmund Optics TechSpec lens with a focal length of $35 \mathrm{~mm}$. The thermographic images were recorded using a FLIR A35 thermographic camera with a sensitivity of $50 \mathrm{mK}( \pm 5 \%)$. Calibration experiments (section 4) were performed with a GE H5K immersion transducer (aperture size 10 $\mathrm{mm}$ ) with a center frequency of $5 \mathrm{MHz}$, and an Onda HGL-0400 lipstick hydrophone (aperture size $400 \mu \mathrm{m}$ ) equipped with an $\mathrm{AH}-2010-100$ pre-amplifier (bandwidth $50 \mathrm{kHz}-100 \mathrm{MHz}$ ). Hydrophone scans were recorded with a scan line step of $75 \mu \mathrm{m}$, taking about 30 minutes per scan. Finally, determination of the spatial resolution (section 5) of the APL visualization was carried out with a medical phased array (L7-4, ATL, Philips) and a fiber-optics hydrophone (aperture size $10 \mu \mathrm{m}$, dynamic range $10 \mathrm{kPa}$ to $15 \mathrm{MPa}$, frequency range $250 \mathrm{kHz}$ to $50 \mathrm{MHz}$ ) from Precision Acoustics. In all setups except the thermographic one, APL emission was recorded by a LM165M 1.4 MPix CCD camera from Lumenera.

\section{Supporting Information}

Supporting Information is available as a separate document.

\section{Acknowledgements}

SM acknowledges the Research Foundation of Flanders (FWO) for financial support in the form of a SB grant (SB 1S33317N). MK is grateful to the Ghent University Special Research Fund through grant number 01N01719. PFS is grateful to the Ghent University Special Research Fund through the GOA Enclose project. Furthermore, we wish to thank dr. Erik Verboven for sharing his expertise in scanning hydrophone measurements and prof. dr. Matthieu Boone from the UGCT (University of Ghent Centre for X-ray Tomography) for the X-ray measurements, 
supported by a UGent Special Research Fund (BOF.EXP.2017.0007). Finally, we thank Stefaan Broekaert and Olivier Janssens at the department of Solid State Sciences at Ghent University and Gert-Wim Bruggert, Bas Benschop and Martin Bos at the University of Twente for technical support.

The authors declare that there is no conflict of interest.

\section{References}

[1] M. Kersemans, W. Van Paepegem, B. Lemmens, K. Van den Abeele, L. Pyl, F. Zastavnik, H. Sol, J. Degrieck, Exp. Mech. 2014, 54, 1059.

[2] M. Castaings, P. Cawley, R. Farlow, G. Hayward, J. Nondestruct. Eval. 1998, 17, 37.

[3] K. Y. Jhang, Int. J. Precis. Eng. Manuf. 2009, 10, 123.

[4] K. Van Den Abeele, J Acoust Soc Am 2007, 122, 73.

[5] C. Errico, J. Pierre, S. Pezet, Y. Desailly, Z. Lenkei, O. Couture, M. Tanter, Nature 2015, 527, 499.

[6] B. Huang, W. Wang, M. Bates, X. Zhuang, Science 2008, 319, 810.

[7] L. V. Wang, S. Hu, Science 2012, 335, 1458.

[8] P. Beard, Interface Focus 2011, 1, 602.

[9] G. Montaldo, M. Tanter, J. Bercoff, N. Benech, M. Fink, IEEE Trans Ultrason Ferroelectr Freq Contro/2009, 56, 489.

[10] C. L. Moore, J. A. Copel, N Eng/ J Med 2011, 364, 749.

[11] J. Provost, C. Papadacci, J. E. Arango, M. Imbault, M. Fink, J. L. Gennisson, M. Tanter, M. Pernot, Phys Med Bio/ 2014, 59, L1.

[12] M. Tanter, M. Fink, IEEE Trans U/trason Ferroelectr Freq Contro/ 2014, 61, 102.

[13] S. Roovers, T. Segers, G. Lajoinie, J. Deprez, M. Versluis, S. C. De Smedt, I. Lentacker, Langmuir 2019, 35, 10173.

[14] C. Huang, H. Zhang, R. Bai, Acta Pharm Sin B 2017, 7, 447.

[15] H. Tang, Y. Zheng, Y. Chen, Adv Mater 2017, 29, 1604105.

[16] T. Mainprize, N. Lipsman, Y. Huang, Y. Meng, A. Bethune, S. Ironside, C. Heyn, R. Alkins, M. Trudeau, A. Sahgal, J. Perry, K. Hynynen, Sci Rep 2019, 9, 321. 
[17] R. Ashida, K. I. Kawabata, T. Maruoka, K. Yamanaka, H. Yoshikawa, T. Ioka, K. Katayama, S. Tanaka, Ultrasound Med Bio/ 2018, 44, 1031.

[18] K. Christensen-Jeffries, O. Couture, P. A. Dayton, Y. C. Eldar, K. Hynynen, F. Kiessling, M. O'Reilly, G. F. Pinton, G. Schmitz, M. X. Tang, M. Tanter, R. J. G. van Sloun, Ultrasound Med Bio/2020, 46, 865.

[19] T. Deffieux, C. Demene, M. Pernot, M. Tanter, Curr Opin Neurobio/ 2018, 50, 128.

[20] M. L. Oelze, J. Mamou, IEEE Trans Ultrason Ferroelectr Freq Contro/ 2016, 63, 336.

[21] X. Ding, S. C. Lin, B. Kiraly, H. Yue, S. Li, I. K. Chiang, J. Shi, S. J. Benkovic, T. J. Huang, Proc Nat/ Acad Sci U S A 2012, 109, 11105.

[22] A. Franklin, A. Marzo, R. Malkin, B. W. Drinkwater, Applied Physics Letters 2017, 111, 094101.

[23] K. Melde, E. Choi, Z. Wu, S. Palagi, T. Qiu, P. Fischer, Adv Mater 2018, 30, 1704507.

[24] K. C. Neuman, A. Nagy, Nature Methods 2008, 5, 491.

[25] D. Baresch, V. Garbin, Proc Natl Acad Sci U S A 2020, 117, 15490.

[26] T. Neumann, H. Ermert, Ultrasonics 2006, 44 Suppl 1, e1561.

[27] N. Kudo, Ultrasound in Medicine \& Biology 2015, 41, 2071.

[28] K. Melde, T. Qiu, R. Fischer, Applied Physics Letters 2018, 113, 133503.

[29] J. J. Joos, K. Lejaeghere, K. Korthout, A. Feng, D. Poelman, P. F. Smet, Phys Chem Chem Phys 2017, 19, 9075.

[30] Y. H. Lin, Z. T. Zhang, Z. L. Tang, J. Y. Zhang, Z. S. Zheng, X. Lu, Mater. Chem. Phys. 2001, 70, 156.

[31] L. C. V. Rodrigues, H. F. Brito, J. Holsa, R. Stefani, M. C. F. C. Felinto, M. Lastusaari, T. Laamanen, L. A. O. Nunes, J. Phys. Chem. C2012, 116, 11232.

[32] K. Van den Eeckhout, D. Poelman, P. F. Smet, Materials (Basel) 2013, 6, 2789.

[33] K. Van den Eeckhout, P. F. Smet, D. Poelman, Materials 2010, 3, 2536.

[34] K. Van den Eeckhout, P. F. Smet, D. Poelman, Materials 2011, 4, 980.

[35] D. Van der Heggen, J. J. Joos, D. C. Rodriguez Burbano, J. A. Capobianco, P. F. Smet, Materials 2017, 10, 867.

[36] M. Kersemans, P. F. Smet, N. Lammens, J. Degrieck, W. Van Paepegem, Applied Physics Letters 2015, 107, 234102.

[37] J. K. Tsou, J. Liu, A. I. Barakat, M. F. Insana, Ultrasound in Medicine \& Biology 2008, 34, 963. 
[38] T. Zhan, C. N. Xu, O. Fukuda, H. Yamada, C. Li, Ultrason Sonochem 2011, 18, 436.

[39] T. Z. Zhan, C. N. Xu, O. Fukuda, H. Yamada, C. S. Li, IOP Conference Series: Materials Science and Engineering 2011, 18, 212011.

[40] N. Terasaki, H. Yamada, C. N. Xu, Catalysis Today 2013, 201, 203.

[41] J. C. Zhang, C. N. Xu, S. Kamimura, Y. Terasawa, H. Yamada, X. Wang, Opt Express 2013, 21, 12976.

[42] J.-C. Zhang, C.-N. Xu, X. Wang, Y.-Z. Long, Journal of Advanced Dielectrics 2014, 04, 1430003.

[43] Y. Zuo, X. Xu, X. Tao, X. Shi, X. Zhou, Z. Gao, X. Sun, H. Peng, Journal of Materials Chemistry C 2019, DOI: 10.1039/c9tc00641a.

[44] L. Zhang, C. N. Xu, H. Yamada, IOP Conference Series: Materials Science and Engineering 2011, $18,212001$.

[45] J. Botterman, K. Van den Eeckhout, I. De Baere, D. Poelman, P. F. Smet, Acta Materialia 2012, 60, 5494.

[46] B. P. Chandra, V. K. Chandra, P. Jha, Physica B: Condensed Matter 2015, 461, 38.

[47] X. H. Fan, J. C. Zhang, M. Zhang, C. Pan, X. Yan, W. P. Han, H. D. Zhang, Y. Z. Long, X. Wang, Opt Express 2017, 25, 14238.

[48] A. Feng, P. F. Smet, Materials 2018, 11, 484.

[49] L. Liu, C.-N. Xu, A. Yoshida, D. Tu, N. Ueno, S. Kainuma, Advanced Materials Technologies 2018, 1800336.

[50] J. C. Zhang, C. N. Xu, Y. Z. Long, Opt Express 2013, 21, 13699.

[51] J.-C. Zhang, X. Wang, G. Marriott, C.-N. Xu, Progress in Materials Science 2019, DOI: https://doi.org/10.1016/j.pmatsci.2019.02.001.

[52] J. A. Kechele, O. Oeckler, F. Stadler, W. Schnick, Solid State Sciences 2009, 11, 537.

[53] J. L. Qin, H. R. Zhang, B. F. Lei, C. F. Hu, J. F. Li, Y. L. Liu, J. X. Meng, J. Wang, M. T. Zheng, Y. Xiao, J. Am. Ceram. Soc. 2013, 96, 3149.

[54] X. Qin, X. Liu, W. Huang, M. Bettinelli, X. Liu, Chem Rev 2017, 117, 4488.

[55] Z. F. Tong, Z. L. Wei, C. Xiao, J. Solid State Chem. 2017, 248, 68.

[56] L. L. Wang, H. Y. Ni, Q. H. Zhang, F. M. Xiao, J. Alloy. Compd. 2011, 509, 10203.

[57] J. Botterman, K. Van den Eeckhout, A. J. J. Bos, P. Dorenbos, P. F. Smet, Opt. Mater. Express $2012,2,341$. 
[58] P. F. Smet, J. Botterman, K. Van den Eeckhout, K. Korthout, D. Poelman, Optical Materials 2014, 36, 1913.

[59] Z. Xia, Z. Xu, M. Chen, Q. Liu, Dalton Trans 2016, 45, 11214.

[60] L. Wang, R. J. Xie, T. Suehiro, T. Takeda, N. Hirosaki, Chem Rev 2018, 118, 1951.

[61] J. C. Zhang, Y. Z. Long, X. Wang, C. N. Xu, Rsc Advances 2014, 4, 40665.

[62] J. Botterman, J. J. Joos, P. F. Smet, Physical Review B 2014, 90, 085147.

[63] B. Liu, C. S. Shi, M. Yin, L. Dong, Z. G. Xiao, J. Alloy. Compd. 2005, 387, 65.

[64] J. J. Joos, K. Korthout, L. Amidani, P. Glatzel, D. Poelman, P. F. Smet, Phys Rev Lett 2020, 125, 033001.

[65] P. Garcia, J. R. Vaille, D. Benoit, F. Ravotti, L. Artola, B. Sagnes, E. Lorfevre, F. Bezerra, L. Dusseau, IEEE Trans. Nucl. Sci. 2007, 54, 2272.

[66] D. Lapraz, H. Prevost, K. Idri, G. Angellier, L. Dusseau, Phys. Status Solidi A-Appl. Mat. 2006, 203, 3793.

[67] N. Mandlik, P. D. Sahare, M. S. Kulkarni, B. C. Bhatt, V. N. Bhoraskar, S. D. Dhole, Journal of Luminescence 2014, 146, 128.

[68] H. Nanto, F. Endo, Y. Hirai, S. Nasu, S. Taniguchi, N. Takeuchi, J. Appl. Phys. 1994, 75, 7493.

[69] C. Tydtgat, K. W. Meert, D. Poelman, P. F. Smet, Opt. Mater. Express 2016, 6, 844.

[70] S. C. Prashantha, B. N. Lakshminarasappa, B. M. Nagabhushana, J. Alloy. Compd. 2011, 509, 10185.

[71] J. Ueda, P. Dorenbos, A. J. J. Bos, A. Meijerink, S. Tanabe, J. Phys. Chem. C2015, 119, 25003.

[72] H. Uoyama, K. Goushi, K. Shizu, H. Nomura, C. Adachi, Nature 2012, 492, 234.

[73] B. J. Luff, P. D. Townsend, Meas. Sci. Technol. 1993, 4, 65.

[74] N. Salah, P. D. Sahare, S. P. Lochab, P. Kumar, Radiation Measurements 2006, 41, 40.

[75] L. Wang, H. Ni, Q. Zhang, F. Xiao, J. Nanosci. Nanotechnol. 2016, 16, 4008.

[76] X. F. Wang, Q. Liu, Y. Y. Bu, C. S. Liu, T. Liu, X. H. Yan, Rsc Advances 2015, 5, 86219.

[77] F. Vetrone, R. Naccache, A. Zamarrón, A. Juarranz de la Fuente, F. Sanz-Rodríguez, L. Martinez Maestro, E. Martín Rodriguez, D. Jaque, J. García Solé, J. A. Capobianco, ACS Nano 2010, 4, 3254.

[78] R. R. Petit, S. E. Michels, A. Feng, P. F. Smet, Light Sci App/ 2019, 8, 124.

[79] J. E. Kennedy, Nat Rev Cancer 2005, 5, 321. 
[80] A. Blana, B. Walter, S. Rogenhofer, W. F. Wieland, Urology 2004, 63, 297.

[81] A. J. J. Bos, Radiation Measurements 2006, 41, S45.

[82] J. T. Randall, M. H. F. Wilkins, M. L. E. Oliphant, Proceedings of the Royal Society of London. Series A. Mathematical and Physical Sciences 1997, 184, 365.

[83] K. Van den Eeckhout, A. J. J. Bos, D. Poelman, P. F. Smet, Physical Review B 2013, 87, 045126.

[84] J. Botterman, P. F. Smet, Opt Express 2015, 23, A868.

[85] P. F. Smet, K. Van den Eeckhout, A. J. J. Bos, E. van der Kolk, P. Dorenbos, Journal of Luminescence 2012, 132, 682.

[86] V. Pagonis, G. Kitis, C. Furetta, Numerical and Practical Exercises in Thermoluminescence, Springer-Verlag New York, 2006.

[87] Y. Pei, M. Y. Wei, B. Cheng, Y. Liu, Z. Xie, K. Nguyen, B. Yuan, Sci Rep 2014, 4, 4690.

[88] C. Gota, S. Uchiyama, T. Yoshihara, S. Tobita, T. Ohwada, J Phys Chem B 2008, 112, 2829.

[89] K. Martin, R. Fernandez, Ultrasound Med Biol 1997, 23, 1267.

[90] N. Abdullah, A. Abu Talib, A. A. Jaafar, M. A. M. Salleh, W. T. Chong, Experimental Thermal and Fluid Science 2010, 34, 1089. 